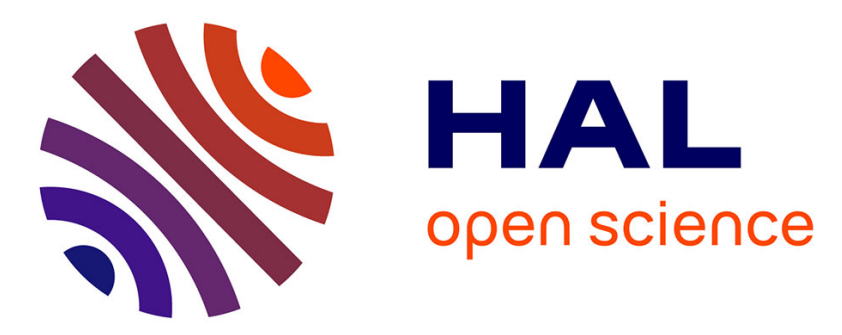

\title{
Dynamical measurements of nucleation and propagation in a domain wall junction at low temperature
}

Stéphane Mangin, G. Marchal, W. Wernsdorfer, A. Sulpice, Klaus Hasselbach, D. Mailly, B. Barbara

\section{- To cite this version:}

Stéphane Mangin, G. Marchal, W. Wernsdorfer, A. Sulpice, Klaus Hasselbach, et al.. Dynamical measurements of nucleation and propagation in a domain wall junction at low temperature. EPL Europhysics Letters, 1997, 39 (6), pp.675. 10.1209/epl/i1997-00414-0 . hal-02104618

\section{HAL Id: hal-02104618 \\ https://hal.univ-lorraine.fr/hal-02104618}

Submitted on 7 May 2019

HAL is a multi-disciplinary open access archive for the deposit and dissemination of scientific research documents, whether they are published or not. The documents may come from teaching and research institutions in France or abroad, or from public or private research centers.
L'archive ouverte pluridisciplinaire HAL, est destinée au dépôt et à la diffusion de documents scientifiques de niveau recherche, publiés ou non, émanant des établissements d'enseignement et de recherche français ou étrangers, des laboratoires publics ou privés. 
Related content

Dynamical measurements of nucleation and propagation in a domain wall junction at low temperature

To cite this article: S. Mangin et al 1997 EPL 39675

View the article online for updates and enhancements.
- Probing domain wall structures in
Co(0001) thin films using ferromagnetic
U. Ebels, P. E. Wigen and K. Ounadjela
- Magnetism of Mn ultra-thin films grown on (001) bcc Fe studied by X-ray magnetic circular dichroism
S. Andrieu, M. Finazzi, F. Yubero et al.
- Oscillation of liquid drops under gravity: Influence of shape on the resonance frequency
M. Perez, Y. Brechet, L. Salvo et al.

\section{Recent citations}

- Relaxation dynamics of magnetization transitions in synthetic antiferromagne with perpendicular anisotropy A Talantsev et al

- Domain wall motion in nanopillar spinvalves with perpendicular anisotropy driven by spin-transfer torques

- Telegraph noise due to domain wall motion driven by spin current in perpendicular magnetized nanopillars J. Cucchiara et al 


\title{
Dynamical measurements of nucleation and propagation in a domain wall junction at low temperature
}

\author{
S. Mangin ${ }^{1,2}$, G. Marchal ${ }^{2}$, W. Wernsdorfer ${ }^{1}$, A. Sulpice ${ }^{3}$ \\ K. Hasselbach ${ }^{3}$, D. Mailly ${ }^{4}$ and B. Barbara ${ }^{1}$ \\ ${ }^{1}$ Laboratoire de Magnétisme Louis Néel, CNRS \\ BP166, 38042 Grenoble Cedex 9, France \\ ${ }^{2}$ Laboratoire de Physique des Matériaux, Université Henri Poincaré-Nancy I \\ BP239, 54506 Vandoeuvre les Nancy Cedex, France \\ ${ }^{3}$ Centre de Recherche sur les Très Basses Températures, CNRS \\ BP166, 38042 Grenoble Cedex 9, France \\ ${ }^{4}$ Laboratoire de Microstructures et Microélectroniques, CNRS \\ 196 Avenue Ravera 92220 Bagneux, France
}

(received 18 November 1996; accepted in final form 29 July 1997)

PACS. 75.60Ch - Domain walls and domain structure.

PACS. 75.70-i - Magnetic films and multilayers.

PACS. $75.45+\mathrm{j}$ - Macroscopic quantum phenomena in magnetic systems.

\begin{abstract}
GdFe $(1000 \AA) / \mathrm{TbFe}(e) / \mathrm{GdFe}(500 \AA)$ sandwiches, where the thickness $e$ of the TbFe film can be as small as $2 \AA$, constitutes a Domain Wall Junction (DWJ). In this device, a $180^{\circ}$ domain wall, nucleated in the thicker GdFe layer, crosses an artificial energy barrier (the $\mathrm{TbFe}$ layer), by thermal activation (above $1 \mathrm{~K}$ ). DWJs constitute a model system for the study of nucleation, pinning and depinning of a domain wall, in both thermal activation and quantum regimes. In the present case, quantum tunnelling of magnetisation is suggested below $0.7 \mathrm{~K}$.
\end{abstract}

Nucleation, pinning and depinning of domain walls, responsible for hysteresis in ferromagnetic systems, have been discussed for several decades. A Domain Wall Junction (DWJ) is a model system for the study of these phenomena in classical and quantum regimes. It is essentially made of an ultra-thin ferromagnetic layer, in sandwich between two other ferromagnetic layers made of a different material [1]. In order to allow the creation of a well-defined Bloch wall across this structure, ferromagnetic films must have an in-plane easy anisotropy direction. Furthermore, the film in sandwich must have a larger anisotropy energy, in order to act as a potential energy barrier preventing the motion of the Bloch wall [1], [2]. The DWJ is also a candidate for the very challenging development of magnetic devices similar to those of transistors and Josephson junctions (it was noted that the equation of motion of a DWJ is formally the same as the one of a Josephson junction [3]). In these systems, a thin layer acting as a potential barrier separates two regions of low potential. The particles injected in a region of low potential cross the barrier in principle by quantum tunnelling. The mechanisms are well established in systems where the particles are electrons or Cooper

(C) Les Editions de Physique 


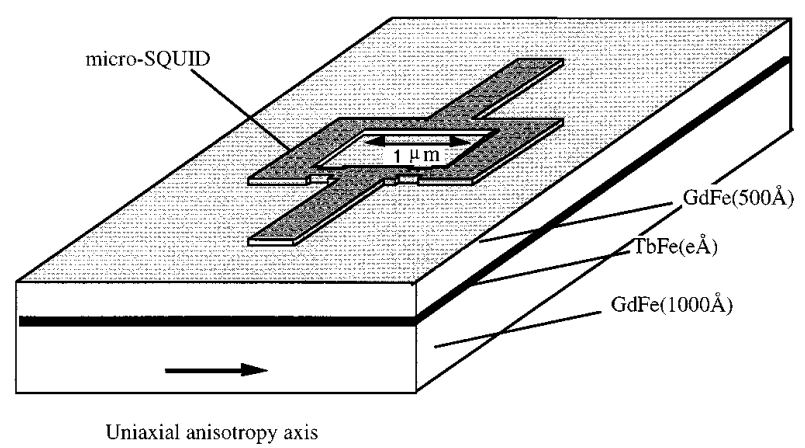

Fig. 1. - Sketch of the sandwich $\operatorname{GdFe}(1000 \AA) / \operatorname{TbFe}(e) / \operatorname{GdFe}(500 \AA)$ sample with the micro-SQUID on the top of it.

pairs. It is still to be demonstrated in DWJ where extended domain walls, although much heavier than electrons $\left(10^{-27} \mathrm{~kg} /(\mathrm{nm})^{2}\right.$ compared to $\left.10^{-30} \mathrm{~kg}\right)$, may behave like such quantum particles. New developments on quantum aspects of DWJ are still rather theoretical [2]-[5]. The number of experimental works devoted to DWJ and other types of tri-layer systems is still very small [6]-[8].

In this letter, we give the first comprehensive study of a DWJ. The system is made of a sandwich of three amorphous ferromagnetic films $\mathrm{GdFe} / \mathrm{TbFe} / \mathrm{GdFe}$ with an in-plane easy anisotropy direction. We show that the central TbFe layer, which can be as thin as $2 \AA$, acts as a barrier and prevents the motion of a Bloch wall, previously nucleated in the thicker GdFe layer. Above $1 \mathrm{~K}$, the barrier can be crossed by thermal activation, but at low temperature anomalies occur which might be attributed to the quantum tunnelling of small domain wall sections. Micro-SQUID experiments show that, at the micrometer scale, a section of domain wall switches across the barrier in a time smaller than the time resolution $\left(10^{-4} \mathrm{~s}\right)$. We have described this switch as a one-dimensional problem applying the Kurkijärvi's model for a Josephson junction [9] to our magnetic case [3], [10].

Elaboration of the DWJ. - The $\mathrm{Gd}_{0.62} \mathrm{Fe}_{0.38}(1000 \AA) / \mathrm{Tb}_{0.45} \mathrm{Fe}_{0.55}(e) / \mathrm{Gd}_{0.62} \mathrm{Fe}_{0.38}(500 \AA)$ tri-layers sketched in fig. 1 were prepared by the evaporation technique in a high vacuum chamber. In order to elaborate the $1000 \AA$ and $500 \AA$ amorphous GdFe layers, gadolinium and iron were simultaneously evaporated from two separate crucibles and deposited on a glass plate first covered by a $100 \AA$ amorphous silicon layer. During the deposition of the amorphous alloy, the substrate was kept at the liquid-nitrogen temperature. The $\mathrm{Tb}_{0.45} \mathrm{Fe}_{0.55}$ layer was prepared in similar conditions. This procedure leads to the occurrence of a spontaneous in-plane anisotropy axis in GdFe layers [11], with an easy axis along the direction perpendicular to that which joins the two sources.

Magnetic characterisation of the DWJ. - The anisotropy constant $K=\frac{M H_{K}}{2} \approx 4.2$. $10^{3} \mathrm{~J} / \mathrm{m}^{3}$ of a $1000 \AA \mathrm{Gd}_{0.62} \mathrm{Fe}_{0.38}$ layer was deduced from the anisotropy field $H_{K}=7.0 \mathrm{mT}$. The anisotropy energy of terbium, coming from the anisotropic $4 f$ electron shells, is much larger than the one of gadolinium which is only related to the $5 d$ electron cloud. In the present case we estimate the $\mathrm{Tb}_{0.45} \mathrm{Fe}_{0.55}$ anisotropy energy to about $10^{6} \mathrm{~J} / \mathrm{m}^{3}$. Using Hansen et al. data [12] and Hasegawa's model [13], [14], the exchange constant $A$ is found to be of the same order in GdFe and $\mathrm{TbFe}\left(15 \cdot 10^{-9} \mathrm{~J} / \mathrm{m}\right)$. It follows that the domain wall energy ( $\gamma=4 \sqrt{A K}$, where $K$ is the anisotropy constant in $\mathrm{J} / \mathrm{m}^{3}$ and $A$ is the exchange energy in $\mathrm{J} / \mathrm{m}$ ) 


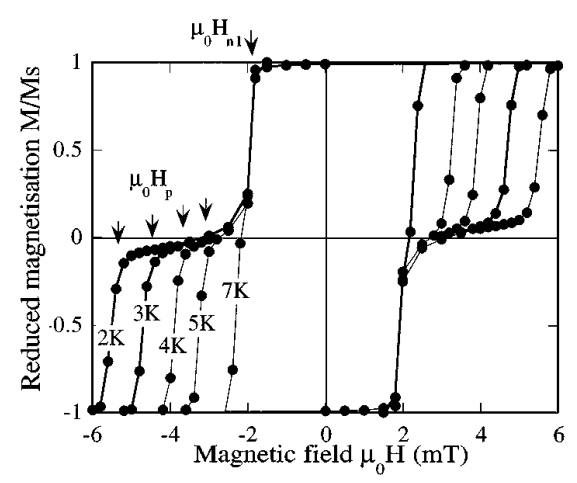

Fig. 2. - Reduced magnetization of a $\operatorname{GdFe}(1000 \AA) / \operatorname{TbFe}(3 \AA) / \operatorname{GdFe}(500 \AA)$ DWJ measured after cooling in a $100 \mathrm{mT}$ field. $H_{\mathrm{n} 1}$ and $H_{\mathrm{p}}$ are, respectively, the nucleation and the propagation fields (quasi-static measurements in a commercial SQUID magnetometer).

is larger in TbFe than in GdFe by an approximated factor of 20. As a consequence, the relative difference of domain wall energies in TbFe and GdFe is $\Delta \gamma / \gamma \approx 20$. In the present case, of a very small thickness $(e)$ of the barrier layer $(\mathrm{TbFe})$, the potential energy barrier width is not the thickness $(e)$ of the layer, but rather the width of the domain wall $\delta=\pi \sqrt{A / K}$ in the rest of the sample (about $700 \AA$ in $\mathrm{GdFe}$ ). This is due to the fact that for $\delta \gg e$, the domain wall averages all the exchange and anisotropy energies over its thickness $\delta$ [1]-[5]. In fact, it starts to interact with the barrier when its front arrives at the position of the TbFe layer and finishes to interact with it when its tail leaves the TbFe layer. Such a domain wall averaging also leads to an energy barrier renormalisation: $(\Delta \gamma / \gamma)_{\text {eff }} \approx(\Delta \gamma / \gamma)(e / \delta) \approx 50(e / \delta)$. This point was developed in a very simple analytical approach in [1], where it was shown that the propagation field $H_{\mathrm{p}}$ of a planar domain wall, through a planar barrier, is given by $M_{\mathrm{s}} H_{\mathrm{p}} \delta=(\Delta \gamma / \gamma)(e / \delta)$.

Experimental procedure. - The injection of a domain wall in the device is realised by the following procedure. First, the sample is saturated along the easy anisotropy axis under a $100 \mathrm{mT}$ applied magnetic field. Then, the field is decreased to zero; the remanent magnetisation is found equal to the saturation magnetisation. Finally, the field is reversed and increased slowly in the negative direction (fig. 2). The application of a field anti-parallel to the magnetisation of the DWJ must create inhomogeneous magnetisation reversals localised on surface defects of the GdFe layers. After some time (corresponding here the field $\mu_{0} H_{\mathrm{n} 1} \approx 2 \mathrm{mT}$ ) these reversed nuclei should rearrange and coalesce giving rise to what we identify as a "homogeneous nucleation" of a $180^{\circ}$ domain wall extending over the whole macroscopic surface of the GdFe layer. This domain wall is blocked by the TbFe layer, the magnetisation of which is at first unchanged. This is shown in fig. 2 by a first drop of the magnetisation. Because of the presence of the domain wall, the amplitude of this drop is smaller than what would be expected from the reversal of the magnetisation of a $1000 \AA \mathrm{GdFe}$ layer. At $H=3.5 \mathrm{mT}, M$ equal to zero, we evaluate a domain wall width of $500 \AA$, which is consistent with $\delta=700 \AA$ in zero field. The difference comes from a compression of the domain wall against the $\mathrm{TbFe}$ layer.

The nucleation described above takes place on the thicker (1000 $\AA$ ) GdFe layer. In the same way, a domain wall could also be homogeneously nucleated from the outer surface of the thinner $(500 \AA) \mathrm{GdFe}$ layer. However, that does not occur below a nucleation field $\mu_{0} H_{\mathrm{n} 2} \approx 6 \mathrm{mT}$, significantly larger than $\mu_{0} H_{\mathrm{n} 1}$. The reason for that will be developed in datail in [15], but can be qualitatively understood if we consider that the thickness of a domain wall has to be reasonably smaller than the layer thickness, in order to develop. If this is not the case (as for 

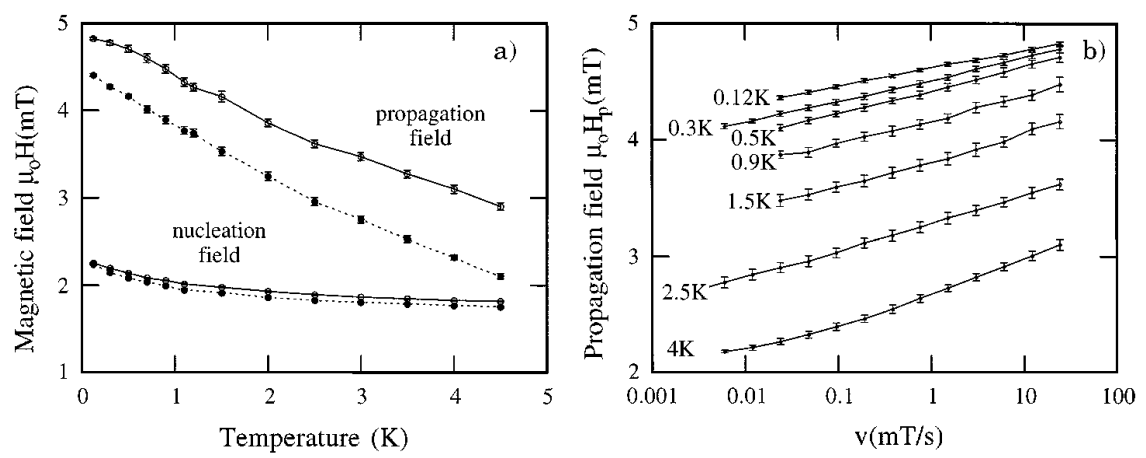

Fig. 3. - a) Temperature dependence of the mean nucleation $\left(H_{\mathrm{n} 1}\right)$ and propagation fields $\left(H_{\mathrm{p}}\right)$ for a $\operatorname{GdFe}(1000 \AA) / \mathrm{TbFe}(2 \AA) / \mathrm{GdFe}(500 \AA) \mathrm{DWJ}$, for two field sweeping rates: $25 \mathrm{mT} / \mathrm{s}$ (full line) and $0.05 \mathrm{mT} / \mathrm{s}$ (dashed line). b) Field sweeping rate dependence of the mean propagation field $H_{\mathrm{p}}$. The width of the nucleation and propagation field distributions are indicated by vertical bars (Micro-SQUID measurements).

the (500 $\AA$ ) GdFe layer) the homogeneous nucleation field becomes very large. Nevertheless, to be sure that the wall propagation through the barrier occurred before the nucleation from the $500 \AA$ A layer, we studied many samples with different thickness. We observed that the field $H_{\mathrm{n} 2}$ increases when the thickness $e_{2}$ decreases. On the other hand, we prepared samples with two TbFe layers $(\operatorname{GdFe}(1000 \AA) / \mathrm{TbFe}(5 \AA) / \operatorname{GdFe}(500 \AA) / \operatorname{TbFe}(10 \AA) / \operatorname{GdFe}(500 \AA))$ and observed the successive crossing of the $\operatorname{TbFe}(5 \AA)$ and $\operatorname{TbFe}(10 \AA)$ layers.

As a consequence, there is a field range in which the wall nucleated in the $1000 \AA$ layer can cross the barrier and continue to propagate into the $500 \AA$ layer before this layer is submitted to a reversal of its magnetisation by nucleation from the outer surface (this range is larger the larger the difference of the GdFe layer thicknesses). It is clear that the crossing of the barrier will be achieved only for well-chosen thicknesses of the TbFe layer and in suitable temperature ranges. When these conditions are fulfilled, we can say that a $180^{\circ}$ domain wall has been injected in the thicker GdFe layer of the device and collected in the thinner GdFe layer.

Dynamical measurements. - The domain wall nucleation and propagation have been studied by magnetisation measurements performed on a commercial SQUID magnetometer above $1.8 \mathrm{~K}$ and on a micro-SQUID [16] stuck on the $\mathrm{GdFe}(1000 \AA) / \mathrm{TbFe}(e) / \mathrm{GdFe}(500 \AA)$ DWJ (see fig. 1), in the temperature range between $0.1 \mathrm{~K}$ and $6 \mathrm{~K}$. The size of the micro-SQUID is $1 \mu \mathrm{m} \times 1 \mu \mathrm{m}$, which provides a local measurement of the flux variations entering the SQUID loop. As the SQUID plane is parallel to the tri-layer, the flux entering the loop is due to magnetic inhomogeneities occurring locally (at different places, over a total scale of about $1 \mu \mathrm{m}$ ) during the magnetisation reversal processes. We observed fast flux fluctuations during the nucleation and the propagation of the domain wall (not time-resolved in $10^{-4} \mathrm{~s}$ ). The switching fields were defined by the maxima of these flux fluctuations. We found that these switching fields were not exactly identical when repeating the hysteresis curves. Therefore, we measured histograms of about 100 switching fields, from which we extracted the mean nucleation field $H_{\mathrm{n} 1}$, the propagation field $H_{\mathrm{p}}$ and the standard deviations of $H_{\mathrm{n} 1}$ and of $H_{\mathrm{p}}$. We measured $H_{\mathrm{n} 1}$ and $H_{\mathrm{p}}$ at temperatures between 0.1 and $4.5 \mathrm{~K}$, for field sweeping rates $\mathrm{d} H / \mathrm{d} t=v$, between 0.01 and $50 \mathrm{mT} / \mathrm{s}$. We discuss here only the results obtained on a tri-layer with a $2 \AA \mathrm{TbFe}$ layer. As expected for thermally activated processes, $H_{\mathrm{n} 1}$ and $H_{\mathrm{p}}$ increase when the temperature is lowered and when the field sweeping rate $v$ is raised (fig. 3 ). 

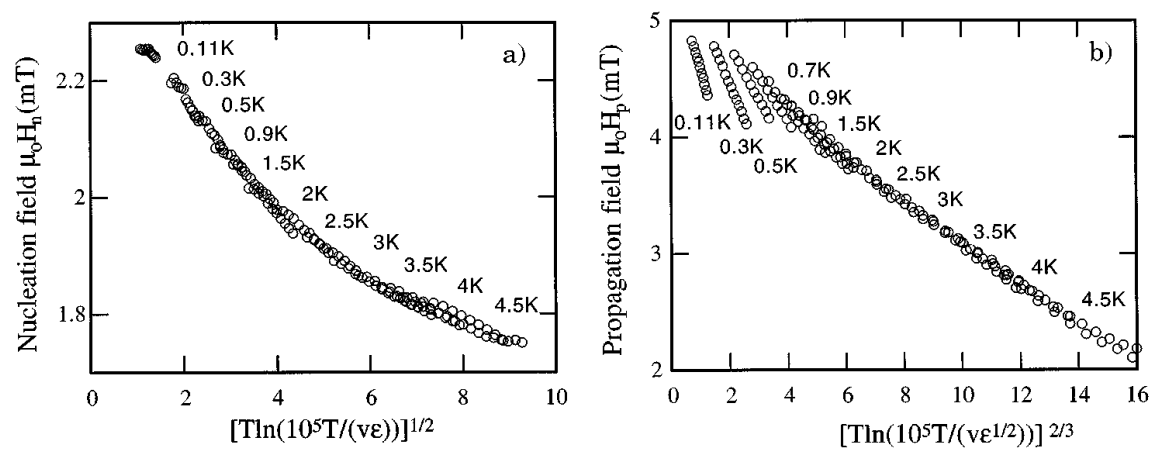

Fig. 4. - Scaling plot of a) the mean nucleation fields $H_{\mathrm{n} 1}(\alpha=2)$ and b) the mean propagation fields $H_{\mathrm{p}}(\alpha=3 / 2)$ for fields sweeping rates between 0.01 and $50 \mathrm{mT} / \mathrm{s}$ and for several temperatures.

Discussion. - The height of the energy barrier is generally given by $E(H)=E_{0}(1-$ $\left.H / H_{0}\right)^{\alpha}=E_{0} \varepsilon^{\alpha}$, where the exponent $\alpha$ depends on the magnetisation reversal process, $E_{0}$ is the height of the energy barrier in zero field and $H_{0}$ is the mean switching field at $T=0 \mathrm{~K}$. It is generally admitted that for a homogeneous $3 \mathrm{D}$ domain wall nucleation $\alpha=2$ and for $2 \mathrm{D}$ domain wall propagation $\alpha=3 / 2$. According to a simple statistical model [3], [9], [10] the mean switching field $\left(H_{\mathrm{n}}\right.$ or $\left.H_{\mathrm{p}}\right)$ should follow the equation

$$
H_{\mathrm{SW}} \cong H_{0}\left(1-\left[\frac{k T}{E_{0}} \ln \left(\frac{c T}{v \varepsilon^{\alpha-1}}\right)\right]^{1 / \alpha}\right)
$$

where $c=k H_{0} /\left(\tau_{0} \alpha E_{0}\right), k$ is the Boltzmann constant and $\tau_{0}$ is inverse of the attempt frequency.

In order to verify the validity of this model, we plotted the mean switching field values as a function of $\left[T \ln \left(c T / v \varepsilon^{\alpha-1}\right)\right]^{1 / \alpha}$. If the underlying model is sufficient, all points should collapse on one master curve by choosing the proper value for the constant $c$. For the right $\alpha$ value the curve is a straight line intercepting the $T=0 \mathrm{~K}$ axis at $H_{0}$ and with an inverses slope equal to $E_{0}$.

For $H_{\mathrm{n}}$, a curvature of the master curves is noticed (fig. 4 a)), which means that a larger $\alpha$ exponent has to be considered. With $\alpha=4$ the linearity is better (giving a mean energy barrier $E_{0}=7800 \mathrm{~K}$ ), but still not good enough. A qualitative explanation of this curvature could be attributed to the existence of localised magnetisation reversals, taking place near the layer surface before the coalescence (see also above). These local reversals being more difficult at low temperature, the "coalescence field" (which we call here "homogeneous nucleation field") will be larger the lower the temperature. In particular, at low temperature, $H_{\mathrm{n}}$ will be larger than predicted by (1), explaining the curvature of fig. $4 \mathrm{a}$ ).

The plot of $H_{\mathrm{p}}$ as a function of $\left[T \ln \left(c T / v \varepsilon^{\alpha-1}\right)\right]^{1 / \alpha}$, with $c \approx 10^{5} \mathrm{mT} / \mathrm{Ks}$ and $\alpha=3 / 2$, is almost linear for $T>1 \mathrm{~K}$ giving $E_{0}=125 \mathrm{~K}$ and $H_{0}=5.5 \mathrm{mT}$ (fig. $4 \mathrm{~b}$ )). A very small curvature is, however, observed which might be inferred to a similar (but much less marked) mechanism as invoked for $H_{\mathrm{n}}$ : at low temperature $H_{\mathrm{p}}$ is slightly larger than predicted by (1), because of larger coupling with adjacent frozen portions of domain wall. At temperature lower than $1 \mathrm{~K}$, the data leave the master curve. That means that the low-temperature behaviour cannot be explained from a purely thermally activated process, at least in the frame of the above model. We checked that no continuous plot could be obtained by changing $\alpha$. 
As a matter of fact, the scaling can be recovered if the effective temperature of the system is assumed to be higher than the measured one, which means that the crossing rate is larger than expected from the only thermal activation. Such a behaviour is suggestive of a macroscopic quantum tunnelling behaviour. This would give a crossover temperature between quantum tunnelling and thermal activation close to $0.7 \mathrm{~K}$. Such a value is expected for the quantum reversal of small entities of $\mathrm{TbFe}$. It is interesting to note that we did not observe a crossover temperature for the nucleation process, nor on Dy-Fe DWJ. A possible alternative explanation is the occurrence of avalanches in the propagation process.

Conclusion. - We presented the first study of a domain wall junction (for a $180^{\circ}$ Bloch wall) which is the formal analogue of a Josephson junction. This is a very challenging device from a basic point of view and for future applications. The prepared samples exhibit well-defined uniaxial anisotropy axis necessary to the formation of a $180^{\circ}$ domain wall. A domain wall is then injected in the thicker layer and collected in the thinner one. We established that the barrier, due to a $2 \AA$ thick TbFe layer, was crossed by thermal activation down to $1 \mathrm{~K}$ and that below this temperature, a temperature-independent behaviour takes place which could be due to quantum tunnelling of magnetisation or, alternatively, to a dragging effect of neighbouring wall sections. In the present study the area of the DWJ is macroscopic. In the next future lateral dimensions will be reduced in order to avoid wall spreading, decrease the effective wall mass and the avalanche effects.

We are very pleased to thank L. GUNTHER for his interest and the important discussions we had on this subject.

\section{REFERENCES}

[1] Barbara B. and Uehara M., Proceedings of the Rare Earth Conference, Durham, UK (Institute of Physics Conference Series) Vol. 37 (1978) 203.

[2] Stamp P. C. E., Phys. Rev. Lett., 66 (1991) 2802.

[3] Gunther L. and Barbara B., Phys. Rev. B, 49 (1994) 3926.

[4] Chudnovsky E. M., Iglesias O. and Stamp P. C. E., Phys. Rev. B, 46 (1992) 5392.

[5] Tatara G. and Fukuyama H., Phys. Rev. Lett., 72 (1994) 772.

[6] Barbara B., Wegrowe J. E., Sampaio L. C., Nozières J. P., Uehara M., Novak M., Paulsen C. and Tholence J. L., Phys. Scr., 49 (1993) 268; J. Magn. E Magn. Mater., 140 (1995) 1883.

[7] Wegrowe J. E., Ballou R., Barbara B., Sulpice A., Amaral V. S. and Mailly D., Phys. Rev. B, 52 (1995) 3466.

[8] Dieny B., Givord D. and Ndjaka J. M., J. Magn \& Magn. Mater., 93 (1991) 503.

[9] KurkiJärvi J., Phys. Rev. B, 6 (1971) 832.

[10] Garg A., Phys. Rev. B, 51 (1995) 15592.

[11] Mangin S., Bellouard C., Marchal G. and Barbara B., J. Magn. E Magn. Mater., 165 (1997) $161,13$.

[12] Hansen P., Clausen C., Much G., Rosenkranz M. and Witter K., J. Appl. Phys., 66 (1989) 756.

[13] Hasegawa R., J. Appl. Phys., 45 (1989) 3109.

[14] Mimura Y., Imamura N., Kobayashi T., Okada A. and Kushiro Y., J. Appl. Phys., 49 (1978) 1208.

[15] Mangin S., Marchal G. and Barbara B., to be published in Phys. Rev. B.

[16] Wernsdorfer W., Bonet Orozco E., Hasselbach K., Benoit A., Barbara B., Demoncy N., Loiseau A., Boivin D., Pascard H. and Mailly D., Phys. Rev. Lett., 78 (1997) 1791. 